\title{
Il Premio Peano 2013
}

La quattordicesima edizione del Premio Peano ha visto vincitore Cédric Villani con il libro II teorema vivente (Rizzoli, 2013, prima edizione in lingua francese Théorème vivant, Grasset \& Fasquelle, 2012 2). La giuria ristretta, composta da Ferdinando Arzarello, Alberto Conte, Angelo Guerraggio, Franco Pastrone e Federico Peiretti ha confermato il voto espresso dai soci. L'interesse che la Matematica presenta tuttora per molte persone di cultura, dimostrato dal successo di libri, film e opere teatrali dedicate a personaggi vissuti in epoche molto diverse (da Saccheri a Fibonacci, da Gödel a Turing a Nash, per citarne solo alcuni), ci conforta a continuare sulla strada intrapresa con il nostro premio che, nato come piccolo evento, si è ormai affermato a livello internazionale grazie alla scelta dei vincitori, personaggi di primo livello nel modo della Matematica e della divulgazione. I vincitori delle edizioni precedenti, tutti venuti personalmente a Torino per ricevere il premio, sono nomi di grande prestigio sia nel campo della scrittura matematico-letteraria, come Apostolos Doxiadis, che della Matematica militante, come Alain Connes e Gabriele Lolli. Sono state anche premiate "stelle" della divulgazione matematica e scientifica e autori impegnati come Keith Devlin, Mario Livio, Marcus du Sautoy, Peter Pesic, lan Stewart, Donal O'Shea, Ana Millan Gasca e Giorgio Israel, David Ruelle, Benoit Rittaud, Alex Bellos e Peter Higgings.

La premiazione quest'anno avrà luogo, in collaborazione con Centroscienza Onlus, nell'ambito di Giovedi Scienza, al Teatro Colosseo di via Madama Cristina 71 in una data ancora da definire. In tale occasione Cédric Villani riceverà il premio, una targa e terrà una conferenza sul tema del libro vincitore.

\section{Manifesto per un' Furopa di Progresso}

Nei mesi scorsi è stato presentato il "Manifesto per un'Europa di Progresso" che, ispirandosi a illustri precedenti, vuole far sentire la voce degli scienziati sul futuro dell'Europa. Ne riportiamo di seguito un breve estratto rinviando i lettori al sito http://manifestoeuropa.cblue.org dove è possibile sia dare la propria adesione sia partecipare al dibattito su questi temi. "ll mondo è in rapida trasformazione. Società ed economia della conoscenza hanno profondamente ridisegnato equilibri ritenuti consolidati. Aree geografiche depresse hanno conquistato, in tempi storicamente irrisori, potenziali enormi di sviluppo e crescita. Conoscenza, cultura e innovazione rappresentano più che mai il traino decisivo verso il futuro. All'opposto l'Occidente, e alcuni aspetti del suo modello di sviluppo, sono entrati in una crisi profonda. L'Europa, in particolare, risulta investita da gravissimi e apparentemente irresolubili problemi (...). Come scienziate e scienziati di questo continente - consapevoli che esiste un nesso inscindibile tra scienza e democrazia - sentiamo quindi la necessità di metterci in gioco. Di ribadire che il processo di costruzione degli Stati Uniti d'Europa è la più importante opportunità che ci è concessa dalla Storia (...). L'unica ri- sposta possibile alla crisi incombente è allora la costruzione dell'Europa dei popoli, di un'Europa di Progresso! Realizzata sulla base dei principi di libertà, democrazia, conoscenza e solidarietà. Nutriamo la stessa speranza con cui Albert Einstein e Georg Friedrich Nicolai nel "Manifesto agli Europei" del 1914 richiamarono alla ragione i popoli europei contro la sventura della guerra, e con cui Altiero Spinelli, Eugenio Colorni ed Ernesto Rossi ispirarono l'idea d'Europa nel loro "Manifesto di Ventotene" del 1943. Le stesse idee che ebbero indipendentemente fautori illustri anche in tutti i Paesi d'Europa. 